\title{
CHARACTERIZATIONS OF STRICTLY SINGULAR AND STRICTLY COSINGULAR OPERATORS BY PERTURBATION CLASSES
}

\author{
PIETRO AIENA \\ Dipartimento di Metodi e Modelli Matematici, Facoltà di Ingegneria, Università di Palermo, \\ Viale delle Scienze, I-90128 Palermo, Italy \\ E-mail: paiena@unipa.it \\ MANUEL GONZÁLEZ \\ Departamento de Matemáticas, Facultad de Ciencias, Universidad de Cantabria, E-39071 Santander, Spain \\ E-mail: gonzalem@unican.es \\ and ANTONIO MARTÍNEZ-ABEJÓN \\ Departamento de Matemáticas, Facultad de Ciencias, Universidad de Oviedo, E-33007 Oviedo, Spain \\ E-mail: ama@uniovi.es
}

(Received 8 October 2010; accepted 11 April 2011; first published online 2 August 2011)

\begin{abstract}
We consider a class of operators that contains the strictly singular operators $\mathcal{S} \mathcal{S}$ and it is contained in the perturbation class of the upper semi-Fredholm operators $P \Phi_{+}$. We show that this class is strictly contained in $P \Phi_{+}$, solving a question of Friedman. We obtain similar results for the strictly cosingular operators $\mathcal{S C}$ and the perturbation class of the lower semi-Fredholm operators $P \Phi_{-}$. We also characterize $\mathcal{S S}$ in terms of $P \Phi_{+}$and $\mathcal{S C}$ in terms of $P \Phi_{-}$. As a consequence, we show that $\mathcal{S} \mathcal{S}$ and $\mathcal{S C}$ are the biggest operator ideals contained in $P \Phi_{+}$and $P \Phi_{-}$, respectively.
\end{abstract}

2010 Mathematics Subject Classification. 47A53.

1. Introduction. The strictly singular operators $\mathcal{S S}$ were introduced by Kato [14] and he showed that they are contained in the perturbation class of the upper semiFredholm operators $P \Phi_{+}$. It has been a long-standing open problem whether these two classes coincide, until a negative solution was given in [9]. In [7], Friedman considered the following condition (C) for an operator $K \in \mathcal{L}(X, Y)$ :

(C) $S \in \mathcal{L}(X, Y), \operatorname{dim}\left(X^{*} /\left(R\left(K^{*}\right)+R\left(S^{*}\right)\right)\right)<\infty \Rightarrow \operatorname{dim}\left(X^{*} / R\left(S^{*}\right)\right)<\infty$,

where $K^{*}$ is the conjugate operator of $K$. She showed that

$$
K \in \mathcal{S S}(X, Y) \Rightarrow K \text { satisfies }(\mathrm{C}) \Rightarrow K \in P \Phi_{+}(X, Y),
$$

proposing as a question whether condition (C) characterizes the operators in $P \Phi_{+}(X, Y)$. Moreover, she characterized the strictly singular operators in terms of $P \Phi_{+}$.

Supported in part by MICINN (Spain), Grant MTM2010-20190. 
Here, we give a negative answer to Friedman's question and two refinements of her characterization of $\mathcal{S S}$ in terms of $P \Phi_{+}$. As a consequence of the refinement, we show that $\mathcal{S S}$ is the biggest operator ideal contained in $P \Phi_{+}$.

The strictly cosingular operators $\mathcal{S C}$ were introduced by Pełczyński [15], and Vladimirskii [19] showed that they are contained in the perturbation class of the lower semi-Fredholm operators $P \Phi_{-}$. We consider the following condition (D) for an operator $K \in \mathcal{L}(X, Y)$ :

(D) $S \in \mathcal{L}(X, Y), \operatorname{dim}(Y /(R(K)+R(S)))<\infty \Rightarrow \operatorname{dim}(Y / R(S))<\infty$.

We prove that $K \in \mathcal{S C}(X, Y) \Rightarrow K$ satisfies (D) $\Rightarrow K \in P \Phi_{-}(X, Y)$, and show that condition (D) does not characterize the operators in $P \Phi_{-}(X, Y)$. Moreover, we give two characterizations of the strictly cosingular operators in terms of $P \Phi_{-}$and, as a consequence, we show that $\mathcal{S C}$ is the biggest operator ideal contained in $P \Phi_{-}$.

The questions whether conditions (C) and (D) characterize $\mathcal{S} \mathcal{S}$ and $\mathcal{S C}$, respectively, remain open. We observe that to obtain negative answers we would need new counterexamples to the perturbation classes problem. Indeed, $\mathcal{S} \mathcal{S}(X, Y)=$ $P \Phi_{+}(X, Y)$ implies that condition $(\mathrm{C})$ characterizes $\mathcal{S} \mathcal{S}$ and $P \Phi_{+}$for operators in $\mathcal{L}(X, Y)$, and the same happens with $\mathcal{S C}, P \Phi_{-}$and condition (D). We refer to [9] and $[\mathbf{8}]$ for two counterexamples and to $[3, \mathbf{8}, \mathbf{1 1}, \mathbf{1 2}]$ for recent positive answers to the perturbation classes problem.

For $X, Y$ Banach spaces, we denote by $\mathcal{L}(X, Y)$ the set of (continuous linear) operators from $X$ into $Y$, and for $T \in \mathcal{L}(X, Y), T^{*} \in \mathcal{L}\left(Y^{*}, X^{*}\right)$ is the conjugate operator of $T$. An operator $T \in \mathcal{L}(X, Y)$ is upper semi-Fredholm if its kernel $N(T)$ is finite dimensional and its range $R(T)$ is closed; and $T$ is lower semi-Fredholm if its range is finite codimensional; hence, closed by [18, Theorem IV.5.10]. We denote respectively by $\Phi_{+}$and $\Phi_{-}$the classes of all upper semi-Fredholm and lower semiFredholm operators. It follows from the basic duality relations for operators that $T \in \Phi_{+}$if and only if $T^{*} \in \Phi_{-}$and $T \in \Phi_{-}$if and only if $T^{*} \in \Phi_{+}$. The class of Fredholm operators is $\Phi:=\Phi_{+} \cap \Phi_{-}$. Given a class $\mathcal{A}$ of operators, we denote

$$
\mathcal{A}(X, Y):=\{T \in \mathcal{L}(X, Y): T \in \mathcal{A}\}
$$

and we write $\mathcal{A}(X)$ in the case $X=Y$.

Given a closed subspace $M$ of $X$, let us denote by $J_{M}$ the inclusion of $M$ into $X$, and by $Q_{M}$ the quotient map from $X$ onto $X / M$. Recall that an operator $T \in \mathcal{L}(X, Y)$ is said to be strictly singular if a restriction $T J_{E}$ to a closed subspace $E$ is an isomorphism only if $E$ is finite dimensional; $T$ is said to be strictly cosingular if for every closed subspace $F$ of $Y$ the composition $Q_{F} T$ is surjective only if $F$ is finite codimensional; and $T$ is said to be inessential if $I_{X}-S T \in \Phi(X)$ for every $S \in \mathcal{L}(Y, X)$. We denote respectively by $\mathcal{S S}, \mathcal{S C}$ and $\mathcal{I} n$ the classes of strictly singular, strictly cosingular and inessential operators. It is easy to show that $T^{*} \in \mathcal{S S} \Rightarrow T \in \mathcal{S C}$ and $T^{*} \in \mathcal{S C} \Rightarrow T \in \mathcal{S S}$, but the converse implications do not hold [16, Examples 1 and 2].

Let $\mathcal{A}$ be any of the classes $\Phi_{+}, \Phi_{-}$or $\Phi$. The perturbation class of $\mathcal{A}$ is defined by its components:

$$
P \mathcal{A}(X, Y):=\{K \in \mathcal{L}(X, Y): K+T \in \mathcal{A}(X, Y) \text { for all } T \in \mathcal{A}(X, Y)\},
$$

when $\mathcal{A}(X, Y)$ is non-empty. 
The components $P \mathcal{A}(X, Y)$ have been studied by many authors, but there are no good descriptions of them in general [2]. As we observed before, $\mathcal{S S}$ is contained in $P \Phi_{+}, \mathcal{S C}$ is contained in $P \Phi_{-}$and both $P \Phi_{+}$and $P \Phi_{-}$are contained in the ideal $\mathcal{I} n$ of inessential operators, which coincides with $P \Phi$. We refer to [1] and [10] for an exposition of these facts.

In $[17,26.6 .12]$, it was observed that the equality $P \Phi_{+}=\mathcal{S S}$ (or $P \Phi_{-}=\mathcal{S C}$ ) holds if and only if the components $P \Phi_{+}(X)$ (or $P \Phi_{-}(X)$ ) determine an operator ideal. Therefore, the examples in [9] show that neither $P \Phi_{+}$nor $P \Phi_{-}$are operator ideals; i.e., they fail the compatibility conditions stated in $[\mathbf{1 7}, 1.1 .3]$.

2. On Friedman's condition. Recall that $K \in \mathcal{L}(X, Y)$ satisfies condition (C) if

$$
S \in \mathcal{L}(X, Y), \operatorname{dim}\left(X^{*} /\left(R\left(K^{*}\right)+R\left(S^{*}\right)\right)\right)<\infty \Rightarrow \operatorname{dim}\left(X^{*} / R\left(S^{*}\right)\right)<\infty .
$$

Next we give a reformulation of this condition, which is easier to compare with the definitions of $\mathcal{S S}$ and $P \Phi_{+}$.

Given two operators $S, T \in \mathcal{L}(X, Y)$, we consider the operator $(S, T) \in \mathcal{L}(X, Y \times$ $Y)$ defined by $(S, T) x:=(S x, T x)$. Its conjugate operator $(S, T)^{*} \in \mathcal{L}\left(Y^{*} \times Y^{*}, X^{*}\right)$ is given by $(S, T)^{*}(g, h)=S^{*} g+T^{*} h$.

Proposition 2.1. An operator $K \in \mathcal{L}(X, Y)$ satisfies condition $(C)$ if and only if for every $S \in \mathcal{L}(X, Y),(S, K) \in \Phi_{+}$implies $S \in \Phi_{+}$.

Proof. Suppose that $K$ satisfies condition $(C)$ and $(S, K) \in \Phi_{+}(X, Y \times Y)$. Then $(S, K)^{*} \in \Phi_{-}$; hence, its range $R\left((S, K)^{*}\right)=R\left(S^{*}\right)+R\left(K^{*}\right)$ is finite codimensional. Thus, $R\left(S^{*}\right)$ is finite codimensional by condition (C), and [18, Theorem IV.5.10] implies that $R\left(S^{*}\right)$ is closed. Thus, $S^{*} \in \Phi_{-}$and hence $S \in \Phi_{+}$.

Conversely, suppose that for every $S \in \mathcal{L}(X, Y),(S, K) \in \Phi_{+}$implies $S \in \Phi_{+}$. If $R\left(K^{*}\right)+R\left(S^{*}\right)$ is finite codimensional, then $(S, K)^{*} \in \Phi_{-}$, hence $(S, K) \in \Phi_{+}$. Our hypothesis implies $S \in \Phi_{+}$; hence $R\left(S^{*}\right)$ is finite codimensional.

The previous characterization allows us to give an easier proof of the following result of Friedman.

Proposition 2.2. [7, Theorems 3 and 4] For an operator $K \in \mathcal{L}(X, Y)$,

$$
K \in \mathcal{S S} \Rightarrow K \text { satisfies }(C) \Rightarrow K \in P \Phi_{+} .
$$

Proof. For the first implication, note that $K \in \mathcal{S S}(X, Y)$ implies $(0,-K) \in$ $\mathcal{S S}(X, Y \times Y)$, because $\mathcal{S} \mathcal{S}$ is an operator ideal [17, 1.9.4 Theorem]. Thus, $(S, K) \in$ $\Phi_{+}(X, Y \times Y)$ implies $(S, 0)=(S, K)+(0,-K) \in \Phi_{+}$; hence $S \in \Phi_{+}$.

For the second implication, suppose that $K$ satisfies (C) and $S \in \Phi_{+}(X, Y)$. Then $S^{*} \in \Phi_{-}$; hence $R\left(S^{*}+K^{*}\right)+R\left(K^{*}\right)$ is finite codimensional. By condition $(\mathrm{C}), R\left(S^{*}+\right.$ $\left.K^{*}\right)$ is finite codimensional; hence $(S+K)^{*} \in \Phi_{-}$and $S+K \in \Phi_{+}$.

The following example shows that the answer to Friedman's question in [7, p. 350] is negative.

ExAmPLE 2.3. Let $Z$ be the infinite dimensional, reflexive, hereditarily indecomposable Banach space constructed in [13] and let $M$ be a closed subspace 
of $Z$ with

$$
\operatorname{dim} M=\operatorname{dim} Z / M=\infty .
$$

Denoting by $J: M \rightarrow Z$ the inclusion, we consider the operator $K: M \times Z \rightarrow M \times Z$ defined by $K(m, z):=(0, J m)$.

It was proved in [9] that $K \in P \Phi_{+}(M \times Z)$. Let us apply Proposition 2.1 to show that $K$ does not satisfy condition $(C)$. Indeed, if we consider the operator $S: M \times Z \rightarrow$ $M \times Z$ defined by $S(m, z):=(0, z)$, then $S \notin \Phi_{+}$but $(S, K) \in \Phi_{+}(M \times Z,(M \times Z) \times$ $(M \times Z))$.

Next we give a dual version of the previous results for $\mathcal{S C}$ and $P \Phi_{-}$. Recall that $K \in \mathcal{L}(X, Y)$ satisfies condition (D) if

$$
S \in \mathcal{L}(X, Y), \operatorname{dim}(Y /(R(K)+R(S)))<\infty \Rightarrow \operatorname{dim}(Y / R(S))<\infty .
$$

Given two operators $S, T \in \mathcal{L}(X, Y)$, we consider the operator $[S, T] \in \mathcal{L}(X \times$ $X, Y)$ defined by $[S, T](x, z):=S x+T z$.

Proposition 2.4. An operator $K \in \mathcal{L}(X, Y)$ satisfies condition ( $D)$ if and only if for every $S \in \mathcal{L}(X, Y)$, $[S, K] \in \Phi_{-}$implies $S \in \Phi_{-}$.

Proof. Suppose that the operator $K$ satisfies condition (D) and $[S, K] \in \Phi_{-}(X \times$ $X, Y)$. Then $R([S, K])=R(S)+R(K)$ is finite codimensional. Thus, $R(S)$ is finite codimensional by condition (D); hence $S \in \Phi_{-}$.

Conversely, suppose that for every $S \in \mathcal{L}(X, Y),[S, K] \in \Phi_{-}$implies $S \in \Phi_{-}$. If $R(K)+R(S)$ is finite codimensional, then $[S, K] \in \Phi_{-}$. Our hypothesis implies $S \in \Phi_{-}$; hence $R(S)$ is finite codimensional.

The next result is a dual version of [7, Theorems 3 and 4].

Proposition 2.5. For an operator $K \in \mathcal{L}(X, Y)$,

$$
K \in \mathcal{S C} \Rightarrow K \text { satisfies }(D) \Rightarrow K \in P \Phi_{-} .
$$

Proof. For the first implication, note that $K \in \mathcal{S C}(X, Y)$ implies $[0,-K] \in \mathcal{S C}(X \times$ $X, Y)$, because $\mathcal{S C}$ is an operator ideal $[\mathbf{1 7}, 1.10 .4$ Theorem $]$. Thus, $[S, K] \in \Phi_{-}(X \times$ $X, Y)$ implies $[S, 0]=[S, K]+[0,-K] \in \Phi_{-}$; hence $S \in \Phi_{-}$.

For the second implication, suppose that $K$ satisfies (D) and $S \in \Phi_{-}(X, Y)$. Then $R(S+K)+R(K)$ is finite codimensional. By condition $(\mathrm{C}), R(S+K)$ is finite codimensional; hence $S+K \in \Phi_{-}$.

The following example shows that the converse to the second implication in the previous result fails.

EXAmPLE 2.6. Let $K$ be the operator in Example 2.3. Then $K^{*} \in P \Phi_{-}(M \times Z)$ but $K^{*}$ does not satisfy condition (D). Indeed, if we consider the operator $S: M \times Z \rightarrow$ $M \times Z$ in Example 2.3, then $S^{*} \notin \Phi_{-}$but $(S, K)^{*}=\left[S^{*}, K^{*}\right] \in \Phi_{-}$. By Proposition $2.4, K^{*}$ does not satisfy condition (D).

The results of this section leave open the following questions.

QUESTION 1. Does condition (C) characterize the strictly singular operators?

QUESTION 2. Does condition (D) characterize the strictly cosingular operators? 
Our impression is that the answer to both questions should be negative. However, we observe that in order to obtain such negative answers we would need new counterexamples to the perturbation classes problem different from those in [8] and [9].

3. On strictly singular and strictly cosingular operators. Here we give some characterizations of strictly singular operators and strictly cosingular operators in terms of perturbation classes for semi-Fredholm operators.

Definition 3.1. Let $X$ denote a Banach space. The density character dens $(X)$ of $X$ is the least cardinal $\kappa$ for which $X$ has a dense subset of cardinality $\kappa$.

Given a non-empty set $\Gamma$, we denote by $|\Gamma|$ the cardinal of $\Gamma$ and by $\ell_{\infty}(\Gamma)$ the space of all bounded scalar families $\left(a_{i}\right)_{i \in \Gamma}$ with index in $\Gamma$. Note that $\ell_{\infty}(\Gamma)$, endowed with the supremum norm $\left\|\left(a_{i}\right)_{i \in \Gamma}\right\|_{\infty}:=\sup _{i \in \Gamma}\left|a_{i}\right|$, is a Banach space.

Let $\Gamma$ be a set satisfying $|\Gamma| \geq \operatorname{dens}(X)$. Then there is a natural isometric embedding of $X$ into $\ell_{\infty}(\Gamma)$. Indeed, let $\left\{x_{i}: i \in \Gamma\right\}$ be a dense subset of $X$. For each $i \in \Gamma$ we select a norm-one $f_{i} \in X^{*}$ such that $f_{i}\left(x_{i}\right)=\left\|x_{i}\right\|$. Clearly,

$$
J(x):=\left(f_{i}(x)\right)_{i \in \Gamma} \quad \text { for all } x \in X
$$

defines an isometric embedding $J: X \longrightarrow \ell_{\infty}(\Gamma)$. In particular, $J \in \Phi_{+}\left(X, \ell_{\infty}(\Gamma)\right)$.

The following result improves [7, Theorem 6].

THEOREM 3.2. For $T \in \mathcal{L}(X, Y)$, the following statements are equivalent:

(i) $T \in \mathcal{S S}(X, Y)$;

(ii) given $Z$ for which $\Phi_{+}(X, Z) \neq \emptyset, S T \in P \Phi_{+}(X, Z)$ for every $S \in \mathcal{L}(Y, Z)$;

(iii) given a set $\Gamma$ satisfying $|\Gamma| \geq \operatorname{dens}(X), S T \in P \Phi_{+}\left(X, \ell_{\infty}(\Gamma)\right)$ for every $S \in$ $\mathcal{L}\left(Y, \ell_{\infty}(\Gamma)\right)$.

Proof. (i) $\Rightarrow$ (ii) Note that $T \in \mathcal{S} \mathcal{S}(X, Y)$ and $S \in \mathcal{L}(Y, Z)$ imply $S T \in \mathcal{S} \mathcal{S}(X, Z)$, and $\mathcal{S S}(X, Z) \subseteq P \Phi_{+}(X, Z)$.

(ii) $\Rightarrow$ (iii) Observe that $|\Gamma| \geq \operatorname{dens}(X)$ implies $\Phi_{+}\left(X, \ell_{\infty}(\Gamma)\right) \neq \emptyset$.

(iii) $\Rightarrow$ (i) Suppose that $T \notin \mathcal{S S}$. Clearly, we can assume $\|T\|=1$. We take an infinite dimensional closed subspace $M$ of $X$ such that $T J_{M}$ is an isomorphism. So there exists a constant $0<C \leq 1$ such that $\|T m\| \geq C\|m\|$ for each $m \in M$.

Let $\Gamma$ be a set satisfying $|\Gamma| \geq \operatorname{dens}(X)$. We consider two disjoint subsets $\Gamma_{1}$ and $\Gamma_{2}$ of $\Gamma$ such that $\left|\Gamma_{1}\right|=\left|\Gamma_{2}\right|=|\Gamma|$ and $\Gamma=\Gamma_{1} \cup \Gamma_{2}$. Clearly we can identify each $\ell_{\infty}\left(\Gamma_{i}\right)$ $(i=1,2)$ with a subspace of $\ell_{\infty}(\Gamma)$. In this way, we get a decomposition $\ell_{\infty}(\Gamma)=$ $\ell_{\infty}\left(\Gamma_{1}\right) \oplus_{\infty} \ell_{\infty}\left(\Gamma_{2}\right)$. Since dens $(T(M)) \leq\left|\Gamma_{1}\right|$, we can take a dense subset $\left\{y_{i}: i \in \Gamma_{1}\right\}$ of $T(M)$ and norm-one elements $g_{i} \in Y^{*}\left(i \in \Gamma_{1}\right)$ such that $g_{i}\left(y_{i}\right)=\left\|y_{i}\right\|$. We take $g_{i}=0$ in $Y^{*}$ for $i \in \Gamma_{2}$ and define

$$
S(y):=\left(g_{i}(y)\right)_{i \in \Gamma} \quad \text { for all } y \in Y .
$$

Then $S \in \mathcal{L}\left(Y, \ell_{\infty}(\Gamma)\right)$ is a norm-one operator with $R(S) \subset \ell_{\infty}\left(\Gamma_{1}\right)$ and $S J_{T(M)}$ is an isometry; thus $\|S T m\| \geq C\|m\|$ for each $m \in M$.

Similarly, since dens $(X / M) \leq\left|\Gamma_{2}\right|$, we can take a dense subset $\left\{z_{i}: i \in \Gamma_{2}\right\}$ of $X / M$ and norm-one elements $h_{i} \in(X / M)^{*}\left(i \in \Gamma_{2}\right)$ such that $h_{i}\left(z_{i}\right)=\left\|z_{i}\right\|$. We take $h_{i}=0$ in 
$(X / M)^{*}$ for $i \in \Gamma_{1}$ and define

$$
U(z):=\left(h_{i}(z)\right)_{i \in \Gamma} \quad \text { for all } z \in X / M .
$$

Then $U \in \mathcal{L}\left(X / M, \ell_{\infty}(\Gamma)\right)$ is an isometric embedding and $R(U) \subset \ell_{\infty}\left(\Gamma_{2}\right)$.

Let us show that $V:=U Q_{M}+S T$ is an isomorphic embedding. Let $x \in X$ with $\|x\|=1$. Then either $\left\|Q_{M} x\right\|=\operatorname{dist}(x, M) \geq C / 3$ or $\left\|Q_{M} x\right\|<C / 3$. In the latter case, we can write $x=m+z$ with $m \in M$ and $\|z\|<C / 3$; hence $\|S T x\| \geq$ $\|S T m\|-\|S T z\|>C / 3$. Therefore,

$$
\|V x\|=\max \left\{\left\|U Q_{M} x\right\|,\|S T x\|\right\} \geq(C / 3)\|x\| \quad \text { for every } x \in X .
$$

Thus, $U Q_{M}+S T \in \Phi_{+}\left(X, \ell_{\infty}(\Gamma)\right)$. Since $U Q_{M} \notin \Phi_{+}\left(X, \ell_{\infty}(\Gamma)\right)$, we conclude $S T \notin$ $P \Phi_{+}\left(X, \ell_{\infty}(\Gamma)\right)$, and the proof is complete.

Corollary 3.3. Let $X$ be a Banach space and let $\Gamma$ be a set with $|\Gamma| \geq \operatorname{dens}(X)$. Then $P \Phi_{+}\left(X, \ell_{\infty}(\Gamma)\right)=\mathcal{S S}\left(X, \ell_{\infty}(\Gamma)\right)$.

Proof. As we mentioned before, $|\Gamma| \geq \operatorname{dens}(X)$ implies that $\Phi_{+}\left(X, \ell_{\infty}(\Gamma)\right)$ is non-empty. Thus $P \Phi_{+}\left(X, \ell_{\infty}(\Gamma)\right)$ is well defined, and the equality follows from the equivalence of (i) and (iii) in Theorem 3.2 for $Y=\ell_{\infty}(\Gamma)$.

REMARK 3.4. We observe that the coincidence of $\mathcal{S S}(X, Y)$ and $P \Phi_{+}(X, Y)$ in some cases is related with the different positions in which a Banach space can be embedded as a subspace of another Banach space, as studied in [6].

Indeed, in the counterexample given in [9] an infinite dimensional, hereditarily indecomposable Banach space $Z$ and a closed subspace $M$ of $Z$ with $\operatorname{dim} M=$ $\operatorname{dim} Z / M=\infty$ were considered. A key fact for the construction is that, in the product space $M \times Z$, the position of $M$ as $M \times 0$ is non-equivalent with the position as a subspace of $0 \times X$.

Conversely, it was proved in [5] that all the closed subspaces of $\ell_{\infty}(\Gamma)$ isomorphic to a Banach space $X$ with $|\Gamma| \geq \operatorname{dens}(X)$ are in equivalent positions, in the sense that given two of them, there exists an automorphism of $\ell_{\infty}(\Gamma)$ that takes one onto the other. This is the reason behind the result in Corollary 3.3.

The following maximality result follows from Theorem 3.2.

PROPOSITION 3.5. The class of strictly singular operators $\mathcal{S} \mathcal{S}$ is the biggest among the operator ideals $\mathcal{A}$ that satisfy $\mathcal{A}(X, Y) \subset P \Phi_{+}(X, Y)$ for every couple of Banach spaces $X, Y$ for which $\Phi_{+}(X, Y)$ is non-empty.

Proof. It is easy to check that the class $P \Phi_{+}$is injective in the sense that given an operator $K \in \mathcal{L}(X, Y)$ and an isomorphic embedding $J \in \mathcal{L}(Y, Z), J K \in P \Phi_{+} \Rightarrow K \in$ $P \Phi_{+}$. Therefore, if an operator ideal $\mathcal{A}$ is contained in $P \Phi_{+}$, the same happens with its injective hull $\mathcal{A}^{i n j}[\mathbf{1 7}, 4.6 .1]$.

Suppose that $\mathcal{A}$ is an injective operator ideal contained in $P \Phi_{+}$and $K \in \mathcal{A}(X, Y)$. Then part (iii) of Theorem 3.2 implies that $K$ is strictly singular.

We consider the space $\ell_{1}(\Gamma)$ of all absolutely summable scalar families $\left(a_{i}\right)_{i \in \Gamma}$ with index in $\Gamma$. Note that $\ell_{1}(\Gamma)$, endowed with the summing norm $\left\|\left(a_{i}\right)_{i \in \Gamma}\right\|_{1}:=\sum_{i \in \Gamma}\left|a_{i}\right|$, is a Banach space.

The unit vector basis of $\ell_{1}(\Gamma)$ is $\left\{e_{j}: j \in \Gamma\right\}$, where $e_{j}=\left(\delta_{i j}\right)_{i \in \Gamma}$. 
Let $\Gamma$ be a set satisfying $|\Gamma| \geq \operatorname{dens}(X)$. Then there is a natural quotient map from $\ell_{1}(\Gamma)$ onto $X$. Indeed, if $\left\{x_{i}: i \in \Gamma\right\}$ is a dense subset of the unit ball $B_{X}$, then

$$
Q\left(\left(a_{i}\right)_{i \in \Gamma}\right):=\sum_{i \in \Gamma} a_{i} x_{i} \text { for all }\left(a_{i}\right)_{i \in \Gamma} \in \ell_{1}(\Gamma)
$$

defines a quotient map $Q: \ell_{1}(\Gamma) \longrightarrow X$. In particular, $Q \in \Phi_{-}\left(\ell_{1}(\Gamma), X\right)$.

THEOREM 3.6. For $T \in \mathcal{L}(X, Y)$, the following statements are equivalent:

(i) $T \in \mathcal{S C}(X, Y)$;

(ii) given $Z$ for which $\Phi_{-}(Z, Y) \neq \emptyset, T S \in P \Phi_{-}(Z, Y)$ for every $S \in \mathcal{L}(Z, X)$;

(iii) given a set $\Gamma$ satisfying $|\Gamma| \geq \operatorname{dens}(Y), T S \in P \Phi_{-}\left(\ell_{1}(\Gamma), Y\right)$ for every $S \in$ $\mathcal{L}\left(\ell_{1}(\Gamma), X\right)$.

Proof. (i) $\Rightarrow$ (ii) Note that $T \in \mathcal{S C}(X, Y)$ and $S \in \mathcal{L}(Z, X)$ imply $T S \in \mathcal{S C}(Z, Y)$, and $\mathcal{S C}(Z, Y) \subseteq P \Phi_{-}(Z, Y)$.

(ii) $\Rightarrow$ (iii) Observe that $|\Gamma| \geq \operatorname{dens}(Y)$ implies $\Phi_{-}\left(\ell_{1}(\Gamma), Y\right) \neq \emptyset$.

(iii) $\Rightarrow$ (i) Suppose that $T \notin \mathcal{S C}$. We take an infinite codimensional closed subspace $N$ of $Y$ such that $Q_{N} T$ is surjective. Thus, $Y=R(T)+N$.

Let $\Gamma$ be a set satisfying $|\Gamma| \geq \operatorname{dens}(Y)$. We consider two disjoint subsets $\Gamma_{1}$ and $\Gamma_{2}$ of $\Gamma$ such that $\left|\Gamma_{1}\right|=\left|\Gamma_{2}\right|=|\Gamma|$ and $\Gamma=\Gamma_{1} \cup \Gamma_{2}$. Clearly, we can identify each $\ell_{1}\left(\Gamma_{i}\right)(i=1,2)$ with a subspace of $\ell_{1}(\Gamma)$. In this way, we get a decomposition $\ell_{1}(\Gamma)=$ $\ell_{1}\left(\Gamma_{1}\right) \oplus_{1} \ell_{1}\left(\Gamma_{2}\right)$.

Since dens $(Y / N) \leq\left|\Gamma_{1}\right|$, we can take a dense subset $\left\{z_{i}: i \in \Gamma_{1}\right\}$ in the unit ball of $Y / N$. Moreover, since $Q_{N} T$ is surjective, we can select a bounded family $\left\{x_{i}: i \in \Gamma_{1}\right\}$ in $X$ such that $Q_{N} T x_{i}=z_{i}$ for each $i \in \Gamma_{1}$. We define $S \in \mathcal{L}\left(\ell_{1}(\Gamma), X\right)$ by $S e_{i}:=x_{i}$ for $i \in \Gamma_{1}$ and $S e_{i}:=0$ for $i \in \Gamma_{2}$.

Similarly, since dens $(N) \leq\left|\Gamma_{2}\right|$, we can take a dense subset $\left\{y_{i}: i \in \Gamma_{2}\right\}$ in the unit ball of $N$ and define $V \in \mathcal{L}\left(\ell_{1}(\Gamma), N\right)$ by $V e_{i}:=0$ for $i \in \Gamma_{1}$ and $V e_{i}:=y_{i}$ for $i \in \Gamma_{2}$. Clearly, $R(V)=N$.

Now, since $Q_{N} T S$ is surjective, $R(T S)+N=Y$. Thus, the operator $U:=J_{N} V+$ $T S$ is surjective. Since $J_{N} V \notin \Phi_{-}\left(\ell_{1}(\Gamma), Y\right)$, we get $T S \notin P \Phi_{-}\left(\ell_{1}(\Gamma), Y\right)$, and the proof is complete.

Corollary 3.7. Let $Y$ be a Banach space and let $\Gamma$ be a set with $|\Gamma| \geq \operatorname{dens}(Y)$. Then $P \Phi_{-}\left(\ell_{1}(\Gamma), Y\right)=\mathcal{S C}\left(\ell_{1}(\Gamma), Y\right)$.

Proof. As we mentioned before, $|\Gamma| \geq \operatorname{dens}(Y)$ implies that $\Phi_{-}\left(\ell_{1}(\Gamma), Y\right)$ is non-empty. Thus, $P \Phi_{-}\left(\ell_{1}(\Gamma), Y\right)$ is well defined, and the equality follows from the equivalence of (i) and (iii) in Theorem 3.6 for $X=\ell_{1}(\Gamma)$.

Similar to Proposition 3.5, we can derive a maximality result from Theorem 3.6.

PROPOSITION 3.8. The class of strictly cosingular operators $\mathcal{S C}$ is the biggest among the operator ideals $\mathcal{A}$ that satisfy $\mathcal{A}(X, Y) \subset P \Phi_{-}(X, Y)$ for every couple of Banach spaces $X, Y$ for which $\Phi_{-}(X, Y)$ is non-empty.

Proof. It is easy to check that the class $P \Phi_{-}$is surjective in the sense that given an operator $K \in \mathcal{L}(X, Y)$ and a surjective operator $Q \in \mathcal{L}(Z, X), K Q \in P \Phi_{-} \Rightarrow K \in$ $P \Phi_{-}$. Therefore, if an operator ideal $\mathcal{A}$ is contained in $P \Phi_{-}$, the same happens with its surjective hull $\mathcal{A}^{\text {sur }}[\mathbf{1 7}, 4.7 .1]$.

Suppose that $\mathcal{A}$ is a surjective operator ideal contained in $P \Phi_{-}$and $K \in \mathcal{A}(X, Y)$. Then part (iii) of Theorem 3.6 implies that $K$ is strictly cosingular. 
Given a semi-Fredholm operator $T \in \Phi_{+}(X, Y) \cup \Phi_{-}(X, Y)$, the index ind $(T)$ of $T$ is defined by

$$
\operatorname{ind}(T):=\operatorname{dim} N(T)-\operatorname{dim} \frac{Y}{R(T)} \in \mathbb{Z} \cup\{ \pm \infty\}
$$

It is well known that the index is constant on the connected components of the semi-Fredholm operators [1]. Therefore, the following weakenings of condition (ii) in Theorems 3.2 and 3.6 imply that $T \in \mathcal{L}(X, Y)$ is inessential:

(a) for every $S \in \mathcal{L}(Y, X), S T \in P \Phi_{+}(X)$;

(b) for every $S \in \mathcal{L}(Y, X), T S \in P \Phi_{-}(Y)$.

Indeed, if $T$ satisfies (a), then $I_{X}-t S T \in \Phi_{+}(X)$ for every $t>0$. Since $I_{X}$ is a Fredholm operator with index equal to 0 , we conclude that for every $S \in \mathcal{L}(Y, X)$, $I_{X}-S T$ is a Fredholm operator; hence $T \in \mathcal{I} n$. For (b) we can give a similar argument.

However, we will give examples below showing that neither of the two conditions is a characterization of the inessential operators. Observe that it is enough to show a Banach space $Z$ for which $\mathcal{I} n(Z) \neq P \Phi_{+}(Z)$ and $\mathcal{I}_{n}\left(Z^{*}\right) \neq P \Phi_{-}\left(Z^{*}\right)$.

Recall that $T \in \mathcal{L}(X, Y)$ is weakly compact if it sends bounded sets into relatively weakly compact subsets, and it is completely continuous if it sends weakly compact sets into norm-compact sets. A Banach space $X$ has the Dunford-Pettis property (in short, $X$ has the $D P P)$ if every weakly compact operator $T \in \mathcal{L}(X, Y)$ is completely continuous. The $C(K)$ spaces, the $L_{1}(\mu)$ spaces and their dual spaces have the DPP. For these results and additional information, we refer to [4].

The following result is essentially known. We give a proof for completeness.

Proposition 3.9. (a) Suppose that $X$ has the DPP. Then every weakly compact operator $U: X \rightarrow Y$ is strictly singular.

(b) Suppose that $Y^{*}$ has the DPP. Then every weakly compact operator $U: X \rightarrow Y$ is strictly cosingular.

Proof. (a) Suppose that $U: X \rightarrow Y$ is weakly compact, $M$ is a closed subspace of $X$ and $U J_{M}$ is an isomorphism. Since $U J_{M}$ is weakly compact, $M$ is reflexive. Therefore, $J_{M}$ is weakly compact and $U$ is completely continuous, which implies that $U J_{M}$ is compact; hence $M$ is finite dimensional; thus $U \in \mathcal{S S}$.

(b) Suppose that $U: X \rightarrow Y$ is weakly compact. Then $U^{*}: Y^{*} \rightarrow X^{*}$ is weakly compact. By part (a), $U^{*}$ is strictly singular; hence $U \in \mathcal{S C}$.

It follows from Proposition 3.9 that the inclusion $i_{1}: L_{2}[0,1] \rightarrow L_{1}[0,1]$ is strictly cosingular because it is weakly compact and $L_{1}[0,1]^{*}$ has the $D P P$. However, it is not strictly singular because it is an isomorphism on the subspace generated by the Rademacher functions $\left(r_{n}\right)$ [4, Theorem 6.2.3]. Note that $\left(r_{n}\right)$ is an orthonormal sequence in $L_{2}[0,1]$.

The inclusion $j_{\infty}: L_{\infty}[0,1] \rightarrow L_{2}[0,1]$ is strictly singular, but not strictly cosingular. Indeed, since $j_{\infty}$ is weakly compact and $L_{\infty}[0,1]$ has the DPP, $j_{\infty} \in \mathcal{S S}$ (see Proposition 3.9). Moreover, $j_{\infty}$ is the conjugate operator of $i_{1}$. Hence, $i_{1} \notin \mathcal{S S} \Rightarrow$ $j_{\infty} \notin \mathcal{S C}$.

Examples 3.10. There exists a Banach space $Z$ and an operator $T \in \mathcal{L}(Z)$ such that $T \in P \Phi_{+}(Z) \backslash \mathcal{I} n(Z)$ and $T^{*} \in P \Phi_{-}\left(Z^{*}\right) \backslash \mathcal{I} n\left(Z^{*}\right)$. 
Let $S: L_{2}[0,1] \rightarrow L_{2}[0,1]$ denote an isomorphism from $L_{2}[0,1]$ onto the subspace generated by the Rademacher functions $\left(r_{n}\right)$.

We take $Z=L_{2}[0,1] \times L_{1}[0,1] \times L_{1}[0,1]$ and define $T \in \mathcal{L}(Z)$ by

$$
T(f, g, h):=\left(0, i_{1} S f, 0\right) .
$$

Note that $Z^{*}=L_{2}[0,1] \times L_{\infty}[0,1] \times L_{\infty}[0,1]$ and the conjugate operator $T^{*} \in$ $\mathcal{L}\left(Z^{*}\right)$ is given by $T^{*}(f, g, h):=\left(S^{*} j_{\infty} g, 0,0\right)$.

Since $\mathcal{I} n$ is an operator ideal, $T \in \mathcal{I} n(Z)$ and $T^{*} \in \mathcal{I} n\left(Z^{*}\right)$. Let us see that $T=$ $I_{Z} T \notin P \Phi_{+}(Z)$ and $T^{*}=T^{*} I_{Z^{*}} \notin P \Phi_{-}\left(Z^{*}\right)$.

We consider the operator $U \in \mathcal{L}(Z)$ defined by $U(f, g, h):=\left(0,-i_{1} S f, \phi\right)$, where $\phi(t):=g(2 t)$ for $0 \leq t \leq 1 / 2$ and $\phi(t):=h(2 t-1)$ for $1 / 2<t \leq 1$.

Clearly, $U \in \Phi_{+}(\bar{Z})$ and $U+T \notin \Phi_{+}(Z)$; hence $T \notin P \Phi_{+}(Z)$. Similarly, $U^{*} \in$ $\Phi_{-}\left(Z^{*}\right)$ and $U^{*}+T^{*} \notin \Phi_{-}\left(Z^{*}\right)$; hence $T^{*} \notin P \Phi_{+}\left(Z^{*}\right)$.

Observe that

- $P \Phi_{+}\left(L_{2}[0,1], L_{1}[0,1]\right)=\mathcal{S S}\left(L_{2}[0,1], L_{1}[0,1]\right)[\mathbf{1 2}$, Theorem 15];

- $P \Phi_{-}\left(L_{\infty}[0,1], L_{2}[0,1]\right)=\mathcal{S C}\left(L_{\infty}[0,1], L_{2}[0,1]\right)[\mathbf{1 2}$, Corollary 17$]$.

\section{REFERENCES}

1. P. Aiena, Fredholm and local spectral theory, with application to multipliers (Kluwer, Dordrecht, 2004).

2. P. Aiena and M. González, On inessential and improjective operators, Studia Math. 131 (1998), 271-287.

3. P. Aiena, M. González and A. Martinón, On the perturbation classes of semi-Fredholm operators, Glasgow Math. J. 45 (2003), 91-95.

4. F. Albiac and N. Kalton, Topics in Banach space theory, Graduate Texts in Math., Vol. 233 (Springer, New York, 2006).

5. A. Avilés, F. Cabello, J. M. F. Castillo, M. González and Y. Moreno, On separably injective Banach spaces. Preprint.

6. J. M. F. Castillo and A. Plichko, Banach spaces in various positions, J. Funct. Anal. 259 (2010), 2098-2138.

7. T. L. Friedman, Relating strictly singular operators to the condition $X<Y \bmod (\mathcal{S}, \mathcal{T})$ and resulting perturbations, Analysis (Munich) 22 (2002), 347-354.

8. J. Giménez, M. González and A. Martínez-Abejón, Perturbation of semi-Fredholm operators on products of Banach spaces, J. Operator Theory (to appear).

9. M. González, The perturbation classes problem in Fredholm theory, J. Funct. Anal. 200 (2003), 65-70.

10. M. González and A. Martínez-Abejón, Tauberian operators, Operator Theory: Advances and Applications, Vol. 194 (Birkhäuser, Basel, 2010).

11. M. González, A. Martínez-Abejón and M. Salas-Brown, Perturbation classes for semiFredholm operators on subprojective and superprojective spaces, Ann. Acad. Sci. Fennicae Math. (to appear).

12. M. González and M. Salas-Brown, Perturbation classes for semi-Fredholm operators in $L_{p}(\mu)$-spaces, J. Math. Anal. Appl. 370 (2010), 11-17.

13. W. T. Gowers and B. Maurey, The unconditional basic sequence problem, J. Amer. Math. Soc. 6 (1993), 851-874.

14. T. Kato, Perturbation theory for nullity, deficiency and other quantities of linear operators, J. d'Analyse Math. 6 (1958), 261-322.

15. A. Pełczyński, On strictly singular and strictly cosingular operators I. Strictly singular and strictly cosingular operators in C(S)-spaces, Bull. Acad. Polon. Sci. 13 (1965), 31-36. 
16. A. Pełczyński, On strictly singular and strictly cosingular operators II. Strictly singular and strictly cosingular operators in L(v)-spaces, Bull. Acad. Polon. Sci. 13 (1965), 37-41.

17. A. Pietsch, Operator ideals (North-Holland, Amsterdam, 1980).

18. A. E. Taylor and D. C. Lay, Introduction to functional analysis, 2nd ed. (Wiley, New York, 1980).

19. J. I. Vladimirskii, Strictly cosingular operators, Soviet Math. Doklady 8(1967), 739-740. 